\title{
Analysis of Anti-Cough Preparations Available In Indian Market
}

\author{
K.Solomon Raju. M. D. \\ * Associate professor, Department of Pharmacology, Guntur Medical College, Guntur.
}

\begin{abstract}
Objectives: To analyze Anti-cough preparations available in Indian market for their formulations, and cost.

Materials and Methods: Anti-cough preparations listed in Drug Today India (2014)were analyzed for their formulations, and cost. Preparations containing Levocetrizine/Cetrizine/Desloratadine+Guaphenesin+Ambroxol+Terbutaline were considered as Accepted formulations. Preparations containing anti-tussives with anti-histamines $/+$ expectorants $/+$ bronchodilators were considered as Irrational formulations. Accepted formulations and irrational formulations were identified. Prices of accepted formulations were compared with that of irrational formulations.

Results: Out of 589 formulations, 116 were accepted formulations and 200 were irrational formulations. Cost of accepted formulations are cheaper than that of irrational formulations.

Conclusion: The drug regulation authorities made the accepted formulations available to majority of population by making them cheap .Need to have stringent vigilance mechanism to control irrational formulations.
\end{abstract}

Key Words: Anti-cough preparations,anti-tussives, , anti-histamines, accepted formulations, expectorants Irrational formulations.

\section{Introduction}

Cough is most common symptom of airway disease. Most common cause of cough is viral infection of upper respiratory tract. Post viral cough is self limiting. Anti-cough preparations are widely using drugs at $\mathrm{OTC}^{1}$.Many preparations came in irrational formulations. Fixed dose combinations of a centrally acting antitussives with bronchodilator or with an anti-histamine have been banned in India, but many are still marketed ${ }^{7}$. Patients are using them liberally, but not getting relief.

$\mathrm{COUGH}$

Cough is a physiologically useful protective reflex that clears the respiratory tract of accumulated mucus and foreign substances ${ }^{2}$.It occurs due to stimulation of mechano, or chemo receptors in throat, respiratory passages or stretch receptors in lungs ${ }^{7}$.

Common causes for cough are laryngeal and pharyngeal infections and neoplasms; tracheobronchial diseases like tracheobronchitis, bronchial asthma, bronchiectasis, bronchogenic carcinoma; lung diseases like pneumonia, tuberculosis, lung abscess, tropical eosinophilia, pulmonary oedema\&infarction, interstial fibrosis; pleural diseases like pleural effusion, pneumothorax; cardiac diseases like LVF, MS, aneurysm of aorta; enlarged mediastinal lymph nodes, mediastinal tumors, wax or FB in the ear, sinusitis, subphrenic or liver abscess $^{3}$.

Gastroesophageal eflux, use of ACEI, smoking are also cause cough ${ }^{1}$.

Cough is mainly two types.1.Productive cough or useful cough: is characterized by the presence of excessive sputum and may be associated with various underlying diseases. eg. chronic bronchitis, bronchiectasis, Tuberculosis,LVF etc. Needs appropriate treatment with antibiotics. Its suppression is not desirable, may even be harmful, except if the amount of expectoration achieved is small compared to the effort of continuous coughing ${ }^{7}$. 2. Non-productive cough or useless cough: is characterized by dry, irritating cough with minimum or absence of sputum. It should be suppressed ${ }^{7}$. Needs anti-tussives for treatment ${ }^{2}$.

Table-1.SPECIFIC TREATMENT APPROACHES TO COUGH ${ }^{7}$

\begin{tabular}{|l|l|l|}
\hline 1 & Upper/lower respiratory tract infection & Appropriate antibiotics \\
\hline 2 & Smoking/chronic bronchitis/ bronchiectasis & Cessation of smoking/avoidance pollutants ,steam inhalation, postural drainage \\
\hline 3 & Pulmonary tuberculosis & Antitubercular drugs \\
\hline 4 & Asthmatic cough & Inhaled beta ${ }_{2}$ agonists/ corticosteroids/ ipratropium \\
\hline 5 & Cough in pulmonary eosinophilia & Diethyl carbamazine citrate, inhaled corticosteroids \\
\hline 6 & Postnasal drip due to sinusitis & Antibiotic, $\mathrm{H}_{1}$ antihistamine, nasal decongestants. \\
\hline 7 & $\begin{array}{l}\text { Post nasal drip due to allergic/perennial } \\
\text { rhinitis }\end{array}$ & $\begin{array}{l}\text { Avoidance of precipitating factors , corticosteroids nasal spray, } \mathrm{H}_{1} \text { anti- } \\
\text { histamines. }\end{array}$ \\
\hline 8 & Gastroesophageal reflux & Bed head elevation, light dinner, diet modification, $\mathrm{H}_{2}$ blockers,PPI,mosapride \\
\hline 9 & ACE inhibitor associated cough & Substitute ACEI by losartan, NSAIDS \\
\hline 10 & Post viral cough & No specific treatment, subsides by itself \\
\hline
\end{tabular}


Anti-Cough Preparations:

These are classified as

1.Mucoregulatory drugs/ Expectorants

1.1. Mucolytic expectorants: Ambroxol, Acetyl cysteine, Carbocysteine, Bromhexine.

1.2 Mucokinetic expectorants: Sodium or potassium citrate, Ammonium chloride, Potassium iodide ,Guaiphenesin, balsum of tolu, Vasaka.

2 .Anti-tussives :

2.1.Directly acting: Codeine, Pholcodeine, Ethylmorphine, Noscapine, Dextromethorphan, Chlophedianol,

2.2 Indirectly acting: Prenoxdiazine, Lozenges, cough drops, glycerine, liquorice, linctuses.

3.Bronchodilators: Salbutamol,Terbutaline.

4.Anti-histamines : Chlorpheniramine, Diphenhydramine, Promethazine, Cetrizine, Levocetrizine, Desloratidine.

i.Expectorants:

Drugs increase the volume or decrease the viscosity of respiratory secretions, facilitate their removal by ciliary action and coughing.

1.1Mucolytic expectorants:

Drugs alter chemical characteristics of sputum to decrease its viscosity and to facilitate its removal by ciliary action or coughing. eg. acetyl cysteine, carbocysteine, bromhexine, ambroxol, dornase alfa ${ }^{1,2}$. They are useful in cough with viscid, tenacious, thick sputum. Common side effects are rhinorrhoea, lacrimation, nausea, gastric irritation, hypersensitivity. Carbocisteine is contraindicated in peptic ulcer patients ${ }^{7}$.

1.2.Mucokinetic expectorants:

They stimulate the flow of respiratory tract secretions by stimulating bronchial secretory cells to increase the volume and ciliary movements to facilitate their removal. eg. Essential olatile oils (oil anise, oil eucalyptus), ammonium chloride, sodium citrate, guaiacol and guaifenesin ${ }^{2}$. They can affect thyroid function on prolonged use. eg. Potassium iodide ${ }^{7}$. Ammonium salts are nauseating, reflexly increasing respiratory secretions ${ }^{7}$.

Expectorants enhance the clearance of mucus, lack of evidence for their efficacy the FDA has removed most expectorants from the market in a review of over the counter drug. With the exception of guaifenesin, no agents approved as expectorants in the U.S ${ }^{1}$. Steam inhalation and proper hydration may be more helpful in clearing airway mucus ${ }^{7}$.

2anti-Tussives:

They decrease the sensitivity of cough center in the CNS and suppress response of the cough center to peripheral stimuli and decrease mucosal secretion ${ }^{5}$. So they suppress cough center and cough reflex. eg. Codeine, Pholcodeine, dextromethorphan, noscapine, pipazethate, benzonatate, prenoxdiazine, honey, liquorice, syrup tolu, syrup vasaka ${ }^{2}$.They aim to control rather than eliminate cough, anti-tussives should be used only for dry nonproductive cough or if cough is unduly tiring, disturbs sleep or is hazardous (hernia, piles, cardiac disease, ocular surgery) ${ }^{7}$. They may cause drowsiness, bronchoconstrition, constipation, vertigo, dizziness, dry mouth. High doses of dextromethorphan causes ataxia,hallucinations ${ }^{7}$.

3bronchodilators

Stimulation of pulmonary receptors can trigger bronchospasm which induce or aggravate cough in individuals with bronchial hyperreactivity. Bronchodilators relieve cough in such individual and improve the effectiveness of cough in clearing secretions by increasing surface velocity of air flow during the act of coughing. They should be used only when an element of bronchoconstriction is present and not routinely. Their fixed dose combinations with anti-tussives are not satisfactory because of differences in time course of action of the components and liability for indiscriminate $\mathrm{use}^{7}$.

\section{Anti-Histamines:}

They competitively antagonize actions of $\mathrm{H}_{1}$ histamine receptors, block histamine induced bronchoconstriction. They afford relief in cough due to their sedative and anti-cholinergic actions, but lack selectively for the cough center ${ }^{4,7}$. eg cetrizine, levocetrizine, chlorphineramine maleate, desloratidine.

5.Approved Formulations ${ }^{6}$ :

DCGI has given approved combinations for the safety of patients and public. They are Anti-histamine with mucolytic expectorants, mucokinetic with mucolytic expectorants, bronchodilator with mucokinetic 
expectorants, Anti-histamine with mocokinetic and mucolytic expectorants. eg. Levocetrizine+Ambroxol, cetrizine+ambroxol,Guaiphenesin+ambroxol,Desloratidine+ambroxol+guaiphenesin,terbutaline+ambroxol.

6.Irrational/Banned Formulations ${ }^{6}$ :

Central Drugs Standard Control Organisation(CDSCO) released a list of banned combinations in its website. Anti-tussives with any other drug is banned. Pharmaceutical industry is preparing many cough syrups with anti-tussive and anti-histamines, anti-tussive and expectorants. .eg.Dextromethophan/codeine phosphate+chlorpheniramine maleate/cetrizine; Dextromethorphan+guaiphenesin+bromhexine.I

\section{Materials And Methods:}

Anti-cough preparations listed in Drug Today India (2014) ${ }^{4}$ were analyzed for their combinations, and cost. Preparations containing Levocetrizine/Cetrizine/Desloratadine+Guaphenesin+Ambroxol+Terbutaline were considered as Accepted formulations by $\mathrm{DCGi}^{6}$. Preparations containing anti-tussives with antihistamines/+expectorants/+bronchodilators were considered as Irrational formulations by $\mathrm{CDSCO}^{6}$. Accepted formulations and irrational formulations were identified. Prices of accepted formulations were compared with that of irrational formulations. Results were tabulated.

\section{Results:}

Out of 589 formulations, accepted formulations were 116(19.6\%) and irrational formulations were 200(33.9\%).Remaining all 273(46.3\%) were mixed combinations. Cost of accepted formulations were cheaper than that of irrational formulations. Out of 116 accepted formulations, bronchodilator and expectorant preparations were 72 (62\%),anti-histamine and expectorant preparations were 44(37.9\%).Out of 200 irrational formulations, Anti-tussive and anti-histamine combinations were 134(67\%), Anti-tussive and anti-histamine and expectorant combinations were 66(33\%).

TABLE 2..ANTI-COUGH FORMULATIONS: ACCEPTABLE VS IRRATIONAL

\begin{tabular}{|l|l|l|l|}
\hline Acceptable $(\mathrm{n}=116)$ & $19.6 \%$ & Irrational $(\mathrm{n}=200)$ & $33.9 \%$ \\
\hline Bronchodilator+Expectorant $(\mathrm{n}=72)$ & $62 \%$ & Anti-tussive+Anti-histamine $(\mathrm{n}=134)$ & $67 \%$ \\
\hline Anti-histamine+expectorant $(\mathrm{n}=44)$ & $37.9 \%$ & Anti-tussive+Anti-histamine+ Expectorants $(\mathrm{n}=66)$ & $33 \%$ \\
\hline
\end{tabular}

\section{Discussion}

Based on results, out of 589 anti-cough formulations, acceptable formulations are116 (19.6\%).Irrational and miscellaneous formulations are 473 (80.3\%).This shows irrational and miscellaneous formulations are dumped in Indian market. If it continues so on ,pubic will suffer with drug induced health hazards in future.

Accepted formulations are only $19.6 \%$.we have to encourage these drugs for safety of patients. As these are cheaper than that of irrational and miscellaneous formulations ,they are available to everyone.

Out of 589 anti-cough formulations, Bronchodilator+Expectorants formulations are $72(12.2 \%)$ and Anti-histamine+expectorants formulations are $44(7.4 \%)$.This shows that acceptable and essential drugs are less in quantity in Indian market. These are to be increased in quantity for increasing population, having allergy and asthma.

\section{Conclusion:}

The drug regulation authorities made the accepted formulations available to majority of population by making them cheap .Need to have stringent vigilance mechanism to control irrational formulations.

\section{References}

[1] Goodman \&Gilman's the pharmacological basis of therapeutics, $12^{\text {th }}$ ed, $1052-1057$

[2] HLSharma,KKsharma ,Principles of Pharmacology, $2^{\text {nd }}$ ed,p650-652

[3] PJMehta'sPractical medicine., $11^{\text {th }}$ ed, p2

[4] Drug today India(2014) January-March 2014,vol.no.II p886-903,p925.

[5] Lippincott'sillustrated reviews, Pharmacology, $3^{\text {rd }}$ ed,p322

[6] www.cdsco.com website for approved and banned drugs.

[7] KDTripathy,Essentials of medical pharmacology,, $7^{\text {th }}$ ed,p218-221 